\title{
Analysis of the Cost of Varying Levels of User Perceived Quality for Internet Access
}

\author{
Ali Adib Arnab ${ }^{1}$, John Schormans ${ }^{2}$, Sheikh Md. Razibulhasan Raj ${ }^{1}$, and \\ Nafi Ahmad ${ }^{2}$ \\ ${ }^{1}$ University of Global Village, Barisal, Bangladesh, \\ adib9877@yahoo.com, \\ ${ }^{2}$ Queen Mary University of London,London, UK
}

\begin{abstract}
Quality of Service (QoS) metrics deal with network quantities, e.g. latency and loss, whereas Quality of Experience (QoE) provides a proxy metric for end-user experience. Many papers in the literature have proposed mappings between various QoS metrics and QoE. This paper goes further in providing analysis for QoE versus bandwidth cost. We measure QoE using the widely accepted Mean Opinion Score (MOS) rating. Our results naturally show that increasing bandwidth increases MOS. However, we extend this understanding by providing analysis for internet access scenarios, using TCP, and varying the number of TCP sources multiplexed together. For these target scenarios our analysis indicates what MOS increase you get by further expenditure on bandwidth. We anticipate that this will be of considerable value to commercial organizations responsible for bandwidth purchase and allocation.
\end{abstract}

Keywords: Mean Opinion Score (MOS), Quality of Experience (QoE),bandwidth, bandwidth cost, Quality of Service (QoS)

\section{Introduction}

Quality of Experience (QoE) has a significant but complex relationship with Quality of Service (QOS) and its underlying factors. Mean Opinion Score (MOS) ranges from 1 to 5 (Bad to Excellent) and represents QoE. Although considerable work has been carried out, in this paper we have considered QOE within a budget with numerous metrics such as PLP, bandwidth, and round trip time, TCP sending rate factor, packet buffer lengths and packet bottleneck capacity. From the curve fitting, we obtained an analytical expression for bandwidth and bandwidth cost. The goodness of fit is obtained from SSE, R Square, Adjacent $\mathrm{R}$ Square and RMSE. Consequently, we found one equation with variable MOS and bandwidth and another one with variable bandwidth and bandwidth cost. The analysis has been performed multiple times for varying the number of TCP sources.

\section{$1.1 \quad$ Objectives}

The major objective of this research is to identify the mathematical relationship between MoS and bandwidth cost. 


\section{Related work}

QoS has major implications from a policy standpoint [9]. At the present time, users acquire Internet service according to a fixed price. A simple monthly subscription fee or price according to the connection time are common. Dial-up modem, Ethernet, cable Ethernet, or DSL are utilized to provide bandwidth. There may be no bandwidth guarantee or only statistical bandwidth guarantees - either one can be offered. Conventional Internet applications mainly utilize TCP as their transport layer protocol. Packet loss is used as a sign of congestion.

As soon as the sender experiences network congestion, TCP responds quickly by reducing its transmission rate multiplicatively. Consequently, the extent of congestion in the network state partially determines the transmission rate of the TCP sender.

The UK is ranked third in terms of the services and metrics included in this analysis followed by France and Germany [13]. The UK has occupied third place in a table of the weighted average and lowest available basket prices across the services which are covered in analyzing bandwidth cost at the varying level. The US is the most expensive regarding both average and lowest available prices across all the services while France is the least expensive. The UK's highest ranking is acquired by the mobile phone service price, triple-play bundle price, in which category the UK is ranked second followed by France. The UK is placed fifth concerning the price required to receive fixed voice services and this is considered the lowest ranking of the UK [14].

\subsection{Abbreviations and Acronyms}

Mean Opinion Score and its indicator Mean Opinion Score is broadly known as MOS, and has become a widespread perceived media quality indicator [12]. MOS (also superfluously known as the MOS score), is one way of classifying the characteristic of a phone call. This score is set out of a five-point scale, shown in Table 1 below: MOS of 4.0 or higher is toll-quality. Once within the

Table 1. MOS Score vs Performance

\begin{tabular}{||cc||}
\hline MOS Score Performance \\
\hline 5 & Excellent \\
4 & Good \\
3 & Fair \\
2 & Poor \\
1 & Bad \\
\hline
\end{tabular}

building, enterprise voice quality patrons generally expect constant quality while employing their telephone [08]. 
Determining PLP and MOS with constant and variable buffer length Our first requirement is to determine PLP and MOS for different numbers of TCP sources, round trip time, bottleneck capacity in packets and buffer length.We take buffer length values from 10 to 1000 [10]

Plotting of relationship between MOS and bandwidth with constant and variable buffer length We obtain a MOS vs bandwidth graph for different different settings of buffer lengths.

bandwidth and bandwidth cost relationship for different parameters Different countries pays different amounts of money for internet access. It largely depends on the internet providers, facilities, internet access availability of networking telecommunication product, infrastructure etc. For example someone in North America may not be paying the same as someone in Africa. We considered values for UK which will give us range of bandwidth and estimated average cost for that bandwidth.

Finding a formula for bandwidth and bandwidth cost From our analysis we obtained graph for bandwidth and bandwidth cost. Our goal is to relate MOS score and bandwidth cost analytically.

Evaluate how much the bandwidth costs to provide a target MOS level The next step was to use existing formulas for PLP and MOS again. Initially we used bottleneck capacity as one of the parameters to determine PLP. We obtained a formula for MOS and bandwidth cost and plotted various bandwidth cost value and evaluated MOS scores against those values. Also, we obtained a similar curve which we previously obtained from MOS vs bandwidth.

Varying number of TCP sources If we want to differentiate for big organizations and small family houses we need to modify our formula for MOS by changing number of TCP sources. In big organizations, the number of TCP sources will be large and for small household it can be much smaller. We modify the number of TCP sources in our initial formula for MOS. We obtain one graph of MOS vs bandwidth cost for a larger number of TCP sources and one graph of MOS vs bandwidth cost for smaller number of TCP sources.

\section{Method}

\subsection{Determining Parameters Value for Experimental Analysis}

An existing formula for PLP in TCP has been used to determine Mean Opinion Score (MOS) [17]. MOS was plotted against bandwidth and bandwidth has 
been plotted against cost. The relationship of MOS and cost has been determined from MOS vs bandwidth and bandwidth vs cost plots.

To determine the values of MOS we require PLP values. According to the analytical expression and performance evaluation of TCP packet loss probability is given by:

$$
P_{i}=\frac{32 N^{2}}{3 b(m+1)^{2}(C \cdot R T T+Q)^{2}}
$$

$\mathrm{N}=$ Number of TCP sources $=50$

$\mathrm{C}=$ Bottleneck capacity in packets per second $=12500$

$\mathrm{b}=$ number packets acknowledged by an ACK packet $=1$

$\mathrm{m}=$ factor by which TCP sending rate is reduced $=1 / 2$

RTT $=$ Round Trip Time $=0.1$ second

$\mathrm{Q}=$ Packet buffer lengths(bits)

According to [10] we can obtain MOS from PLPs shown in equation 2 below:

$$
M O S=1.46 * \exp (\exp (-44 * P L P))+4.14 * \exp (-2.9 * P L P)
$$

Using different buffer length we get values for PLP and MOS (See Table 1). The $\mathrm{Q}$ value has been taken from 10 to 1000 . With the same packet buffer length and increasing bandwidth (bandwidth) 15 Mbps each time, from 15 to 120 we evaluate PLP and MOS. bandwidth and cost pricing is different worldwide. We

Table 2. Q, PLP and MOS value with different $Q$

\begin{tabular}{||ccc||}
\hline Q & PLP & MOS \\
\hline \hline 10 & $7.465263 \mathrm{e}-01$ & $4.750997 \mathrm{e}-01$ \\
100 & $6.503074 \mathrm{e}-01$ & $6.280123 \mathrm{e}-01$ \\
200 & $5.637028 \mathrm{e}-01$ & $8.073142 \mathrm{e}-01$ \\
400 & $4.353297 \mathrm{e}-01$ & $1.171447 \mathrm{e}+00$ \\
600 & $3.462922 \mathrm{e}-01$ & $1.516566 \mathrm{e}+00$ \\
800 & $2.820191 \mathrm{e}-01$ & $1.827308 \mathrm{e}+00$ \\
1000 & $2.341107 \mathrm{e}-01$ & $2.099708 \mathrm{e}+00$ \\
\hline
\end{tabular}

can get an estimation of bandwidth vs cost for United Kingdom which is used as sample data here as a unit of analysis (Ofcom, 2017).

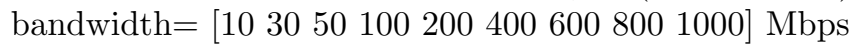

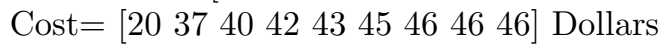


Table 3. Q, bandwidth, PLP and MOS value with same Q

\begin{tabular}{||cccc||}
\hline Q bandwidth & PLP & MOS \\
\hline \hline 10 & 15 & $6.503074 \mathrm{e}-01$ & $6.280123 \mathrm{e}-01$ \\
10 & 30 & $1.753233 \mathrm{e}-01$ & $2.490591 \mathrm{e}+00$ \\
10 & 45 & $7.995852 \mathrm{e}-02$ & $3.326486 \mathrm{e}+00$ \\
10 & 60 & $4.556652 \mathrm{e}-02$ & $3.824152 \mathrm{e}+00$ \\
10 & 75 & $2.939265 \mathrm{e}-02$ & $4.202314 \mathrm{e}+00$ \\
10 & 90 & $2.051913 \mathrm{e}-02$ & $4.492741 \mathrm{e}+00$ \\
10 & 105 & $1.513212 \mathrm{e}-02$ & $4.712481 \mathrm{e}+00$ \\
10 & 120 & $1.161832 \mathrm{e}-02$ & $4.878501 \mathrm{e}+00$ \\
\hline
\end{tabular}

\subsection{Determining formula for bandwidth and bandwidth cost by Curve fitting}

To obtain bandwidth vs cost curve, we need a specific formula for bandwidth and cost.

$$
f(x)=a x^{b}
$$

Coefficients (with $95 \%$ confidence bounds)

$\mathrm{a}=27.13(22.65,31.61)$

$\mathrm{b}=0.0986(0.06944,0.1279)$

The values of $\mathrm{a}$ or $\mathrm{b}$ were provided by the curve fitting as the best fit for the graph we got for bandwidth vs cost. Confidence bounds value are:

Goodness of fit:

SSE: 38.48

R-square 0.9589

Adjusted R-square 0.953

RMSE 2.345

See table IV below: From Table VI we know the method named in curve fit-

Table 4. Goodness of fit parameters for curve fitting

\begin{tabular}{|c|c|c|}
\hline \begin{tabular}{|l|} 
Fit \\
name \\
SSE \\
Adj R- \\
sq
\end{tabular} & $\begin{array}{l}\text { Data } \\
\text { R-square } \\
\text { RMSE }\end{array}$ & $\begin{array}{l}\text { Fit type } \\
\text { DFE } \\
\text { coeff }\end{array}$ \\
\hline \begin{tabular}{|l|} 
Fit 1 \\
38.4806 \\
0.9530
\end{tabular} & $\begin{array}{l}\text { Cost vs bandwidth } \\
0.9589 \\
2.3446\end{array}$ & $\begin{array}{l}\text { Power1 } \\
7 \\
2\end{array}$ \\
\hline
\end{tabular}


Table 5. Method and Algorithm for curve fitting

\begin{tabular}{||cc||}
\hline Method & NonlinearleastSquares \\
\hline \hline Robust & LAR \\
Algorithm & Trust Region \\
DiffMinChange & $1.0 \mathrm{e}-8$ \\
DiffMaxChange & 0.1 \\
MaxFunEvals & 600 \\
Maxlter & 400 \\
TolFun & $1.0 \mathrm{e}-6$ \\
TolX & $1.0 \mathrm{e}-6$ \\
\hline
\end{tabular}

Table 6. Method and Algorithm for curve fitting

\begin{tabular}{|c|c|c|c|}
\hline \multicolumn{4}{|c|}{ Coefficient StartPoint Lower Uppe } \\
\hline $\mathrm{a}$ & 9.2708 & $-\operatorname{lnf}$ & $\operatorname{lnf}$ \\
\hline $\mathrm{b}$ & 0.3019 & $-\operatorname{lnf}$ & $\operatorname{lnf}$ \\
\hline
\end{tabular}

ting is NonlinearleastSquares which is taken automatically by the characteristics, shape and number of squares present in the curve. Among Interpolant, Linear fitting, Polynomial, Rational, Sum of shine, Smoothing Spline, Weibull, Exponential, Gaussian, Fourier we selected Power1 since it gives better visualization and better goodness of fit prediction. The prediction includes calculation of SSE, R-square, Adjacent R-square, RMSE, coefficient with confidence bound. The robust option is enabled and Least Absolute Residuals (LAR) shows more accurate results than Bi Square. LAR mainly focuses on the contrast between residuals rather than squares and Bi-Square reduces weight of the sum of the squares which is necessary for our curve's case.

We will take bandwidth as ' $\mathrm{x}$ ' which lies $\mathrm{X}$ axis and bandwidth cost as ' $\mathrm{f}(\mathrm{x})$ ' which lies in $\mathrm{Y}$ axis. We can acquire values for ' $a$ ' and ' $b$ ' from curve fitting and by implementing the equation (3) we obtain equation (4) below:

$$
\text { Cost }=27.13 * \text { bandwidth } h^{0.0986}
$$

\subsection{Implementing MOS and cost relationship by eliminating bandwidth from bandwidth vs cost formula}

By taking different values of cost we can get different values of bandwidth and if we replace bandwidth from equation (2) we can get different values for cost and MOS. Bottleneck capacity is given by eqn (5) below:

$$
C=\frac{\text { bandwidth } * 1000000}{12000}
$$




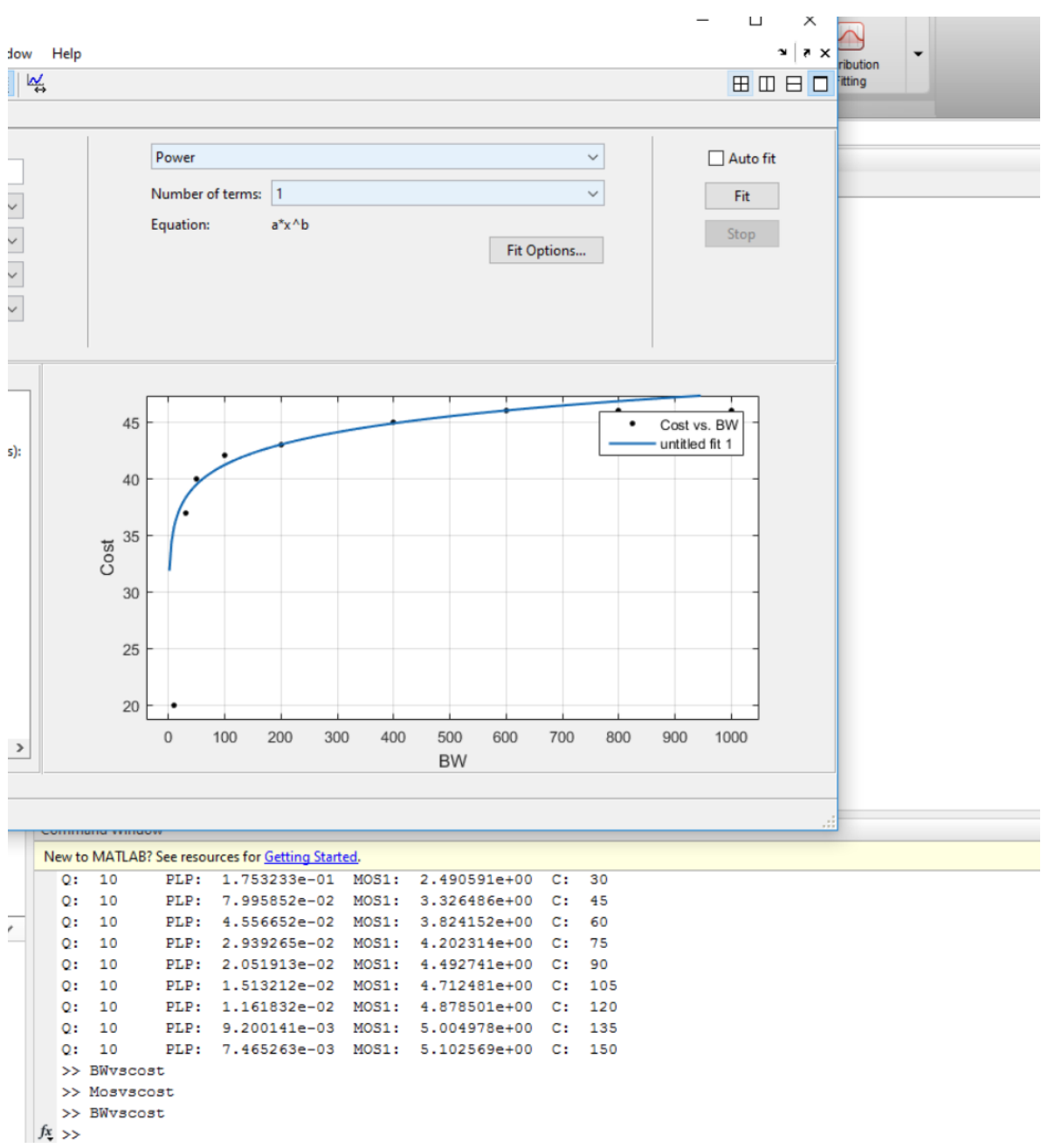

Fig. 1. Setup for applying Curve fitting formula from Cost vs bandwidth to obtain a formula for MOS vs bandwidth cost 


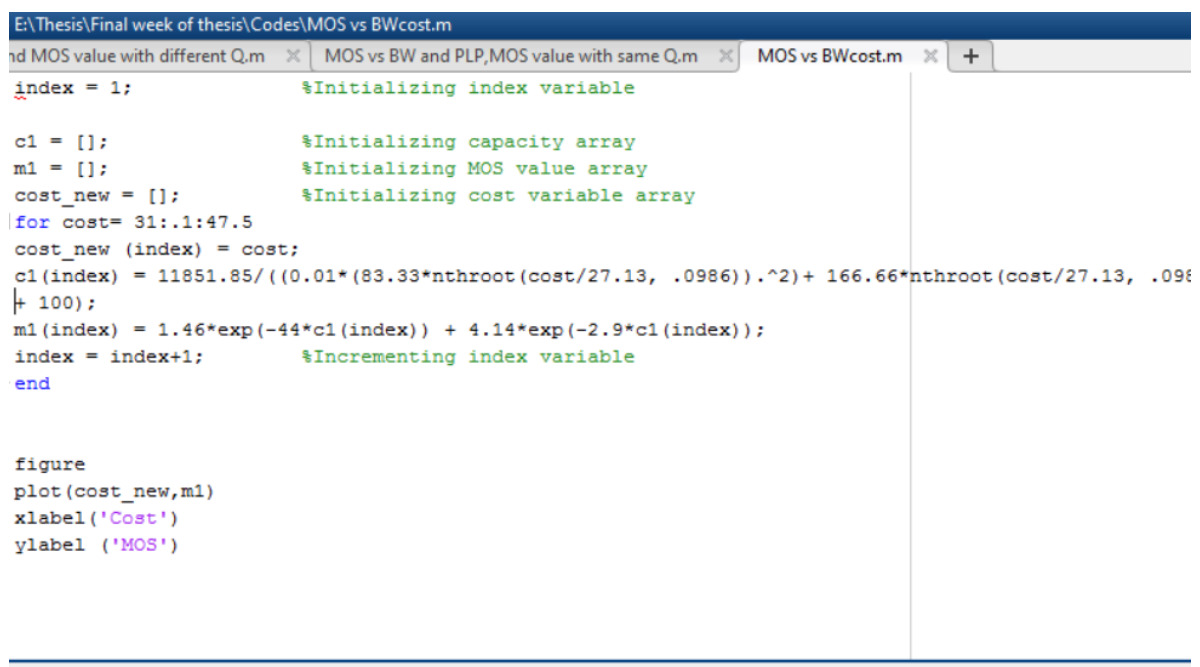

Fig. 2. MOS vs bandwidth cost relationship

MOS and bandwidth From equation (1), (2) and (5) we obtained relationship between MOS and bandwidth which is equation (6):

$$
\begin{aligned}
& \text { MOS }=1.46 e^{-44 . \frac{11851.851}{0.01\left(\frac{B W \cdot 1000}{12}\right)^{2}+2\left(\frac{B W \cdot 1000}{12}\right)+100}} \\
& +4.14 e^{-2.9 \cdot \frac{11851.851}{0.01\left(\frac{B W .1000}{12}\right)^{2}+2\left(\frac{B W .1000}{12}\right)^{2}+100}}
\end{aligned}
$$

MOS and bandwidth Cost If we put bandwidth cost in the place of bandwidth by help of equation (4) we obtain the following relationship which is equation (7):

\subsection{MOS and bandwidth Cost}

To evaluate MOS with different numbers of TCP sources we changed the values for N. We took 2 sample values of N 80 and 500. 80 TCP sources mainly represent a small building or bandwidth use for family purposes. 500 TCP sources represents bigger companies and organizations.

To get MOS and cost relationship, we took a value of $\mathrm{N}=80$ and 500 instead of 


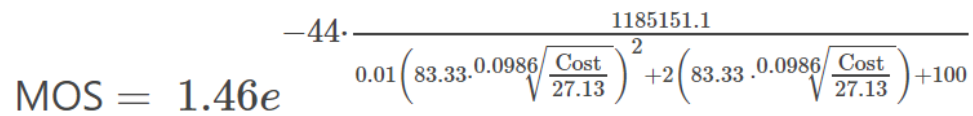

$$
\begin{aligned}
& +4.14 e \\
& -2.9 \cdot \frac{1185151.1}{0.01\left(83.33 \cdot \sqrt[0.0986]{\frac{\text { Cost }}{27.13}}\right)^{2}+2\left(83.33 \cdot \sqrt[0.0986]{\frac{\text { Cost }}{27.13}}\right)+100}
\end{aligned}
$$

50 which provided us a different PLP and a different MOS. the bandwidth and cost relationship remains same as before because it is here seen to have nothing to do with the number of TCP sources. We were able to obtain different MOS and bandwidth formula and different MOS and Cost formula and get output for different number of TCP sources.

Which is denoted by the equation (8): For $\mathrm{N}=500$, MOS and Cost which is

$$
\begin{aligned}
& \text { MOS }=1.46 e^{-44 \cdot \frac{30340.740}{0.01\left(83.33 \cdot \sqrt[0.0986]{\frac{\text { Cost }}{27.13}}\right)^{2}+2\left(83.33 \cdot \sqrt[0.0986]{\frac{\text { Cost }}{27.13}}\right)+100}}
\end{aligned}
$$

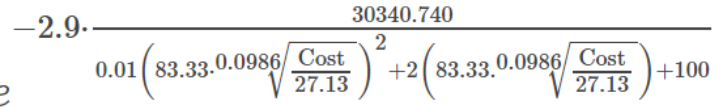

equation (9):

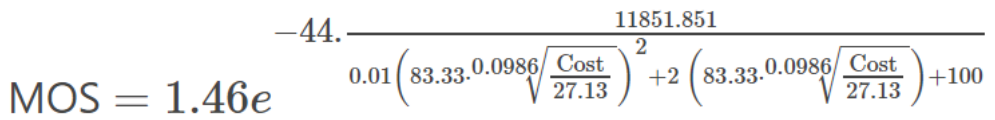

$$
\begin{aligned}
& +4.14 e \\
& -2.9 \cdot \frac{11851.851}{0.01\left(83.33 \cdot \sqrt[0.0986]{\frac{\text { Cost }}{27.13}}\right)^{2}+2\left(83.33 \cdot \sqrt[0.0986]{\frac{\text { Cost }}{27.13}}\right)^{2}+100}
\end{aligned}
$$

\section{Results and Testing}

\subsection{Plotting MOS vs bandwidth (Bps) with different packet buffer lengths}

Our initial formula for PLP provides a relationship between packet loss probability and various parameters including the number of TCP sources, bottleneck capacity, round trip time, number of packets acknowledged by an ACK packet, factor by which TCP sending rate is reduced and packet buffer length. We can 
Ali Adib Arnab et al.

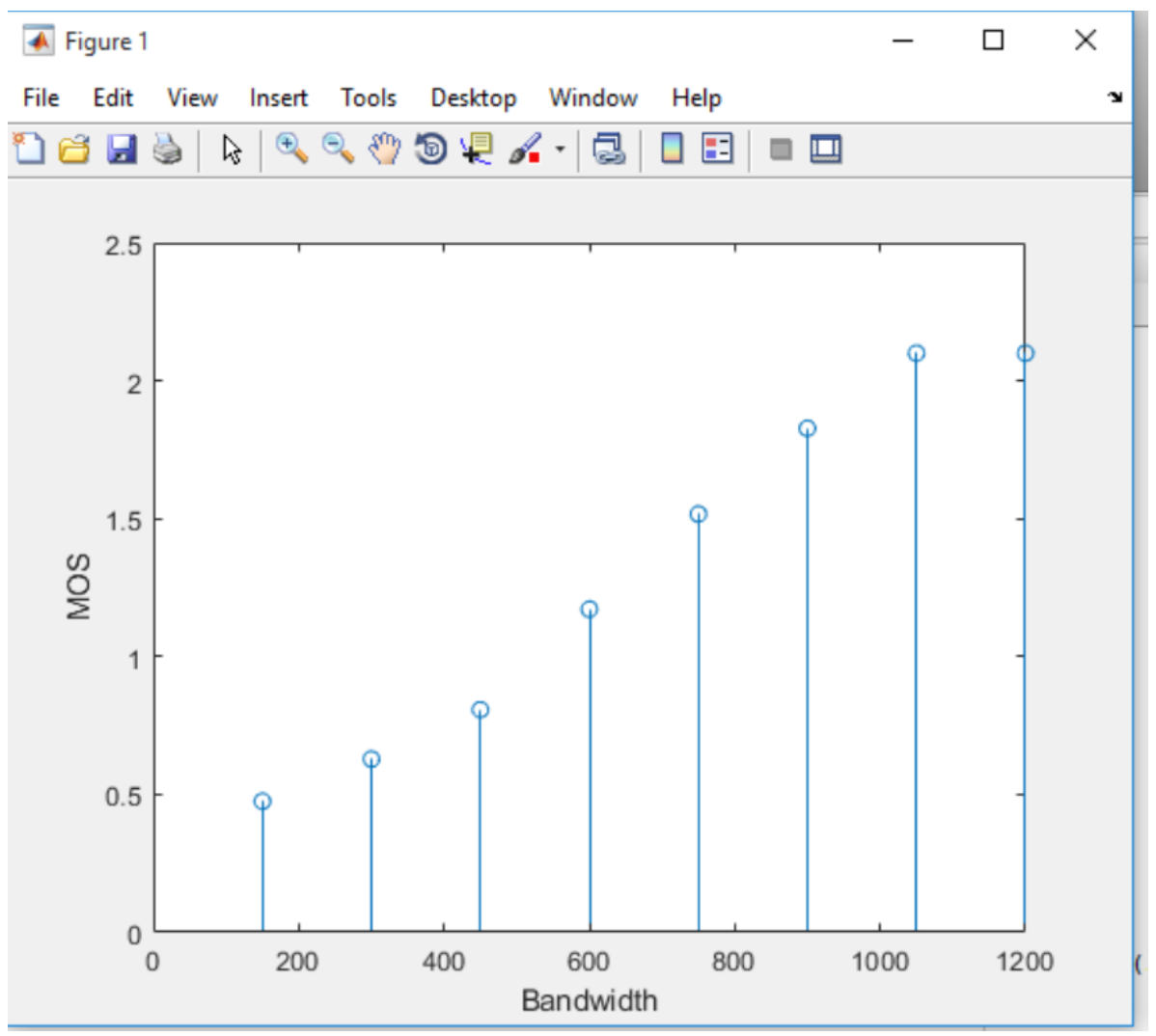

Fig. 3. MOS vs bandwidth (Bps) with different packet buffer lengths 
determine the PLP by taking sample data for this parameter. The MATLAB code and formula for PLP is discussed in 5.1. we then calculated MOS from PLP. Fig 4 is MOS vs bandwidth (Mbps) with different packet buffer lengths.

\subsection{Plotting of MOS vs bandwidth (Mbps) with constant packet buffer length Output}

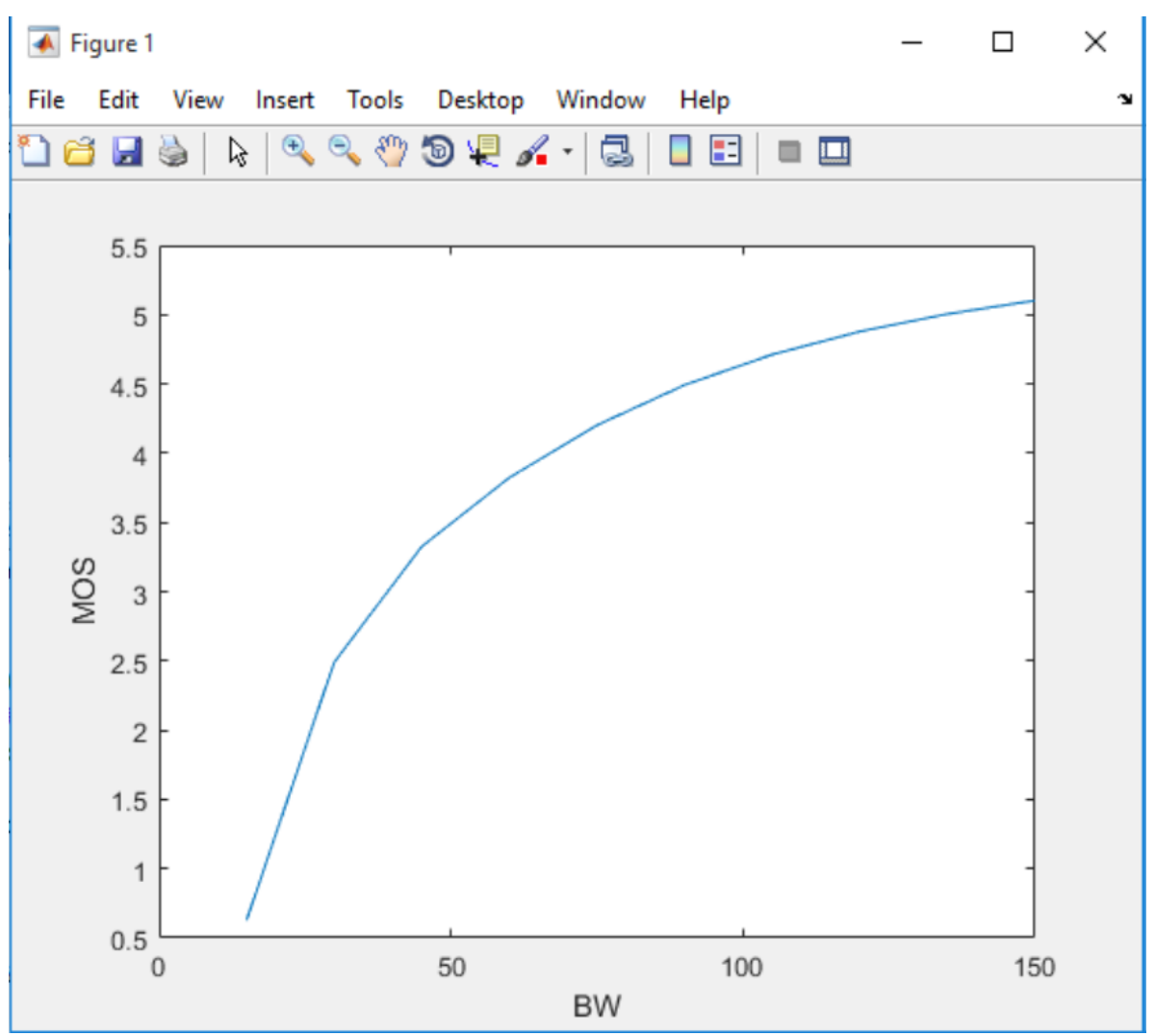

Fig. 4. MOS vs bandwidth (Mbps) with same packet buffer length

If we keep changing buffer length it is very difficult to evaluate MOS change with the effects of bandwidth change. So by keeping the same buffer length, Q which is 10 , we can obtain a MOS vs bandwidth curve. So it is quite evident from Fig (4) that when we increase bandwidth, MOS is also increasing proportionally bandwidth. As a sample when bandwidth is 50 Mbps, MOS is 3.5 (approximately), when bandwidth is $100 \mathrm{Mbps}$ MOS is 4.5 (approximately) and when bandwidth is $150 \mathrm{Mbps}$ MOS is close to 5 . 


\subsection{Plotting of bandwidth Vs bandwidth Cost Relationship}

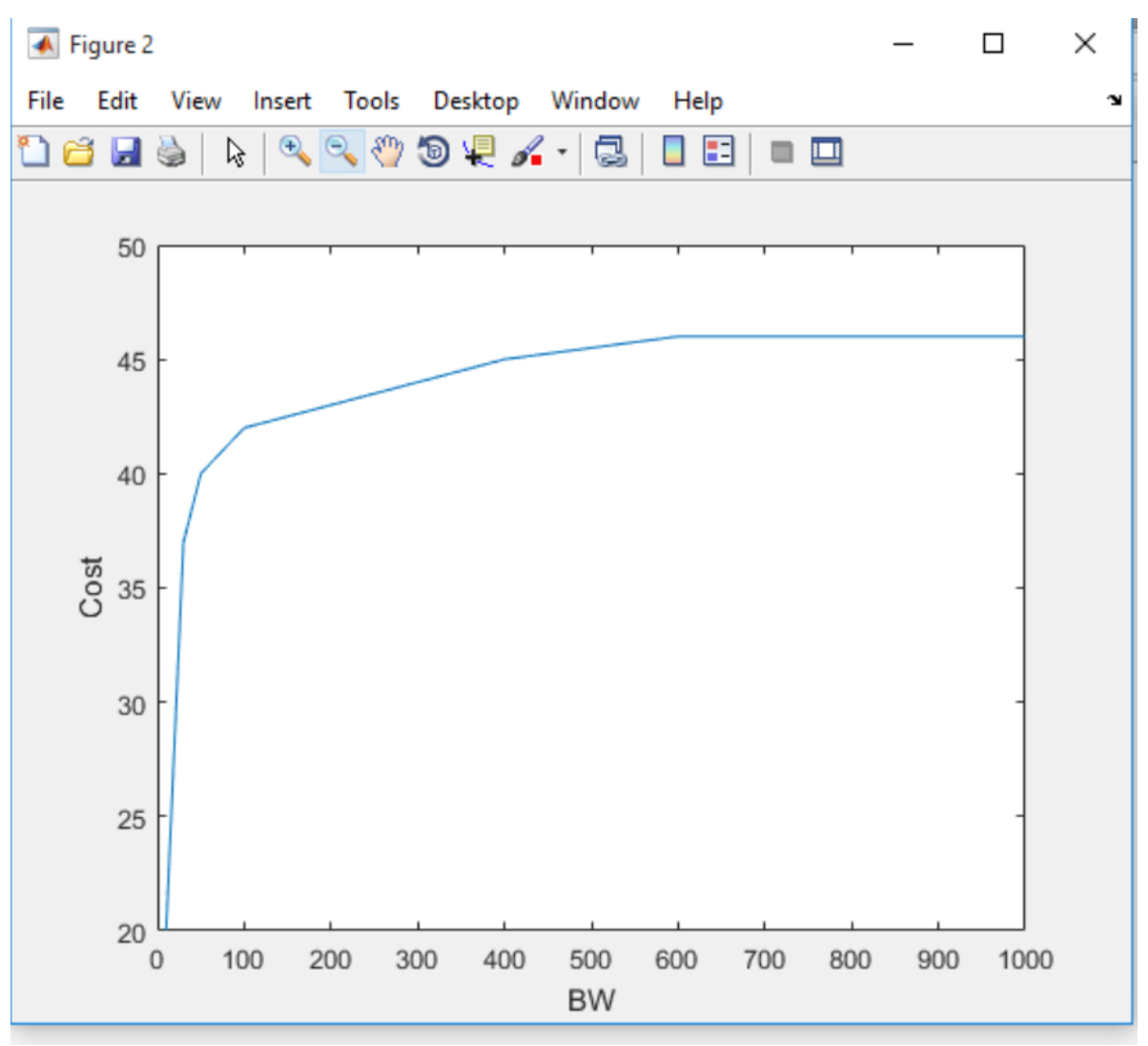

Fig. 5. bandwidth vs bandwidth cost relationship

We took some different parameters for bandwidth cost in the UK. If we look at the figure (5) we can see initially the cost increases within a limited range of bandwidth. In this case even for $20 \mathrm{Mbps}$ the bandwidth price is somewhere close to $35 £$ per month. The rate increases until $100 \mathrm{Mbps}$, from the graph customer has to pay nearly $43 £$ for $100 \mathrm{Mbps}$ per month. Beyond $100 \mathrm{Mbps}$ the cost increment is very slow. From $100 \mathrm{Mbps}$ to $1000 \mathrm{Mbps}$ cost only increases from $43 £$ to $46 £$ per month which is incredibly low. So we can draw conclusions about how much it is worth spending on bandwidth before we run into a law of diminishing returns. 


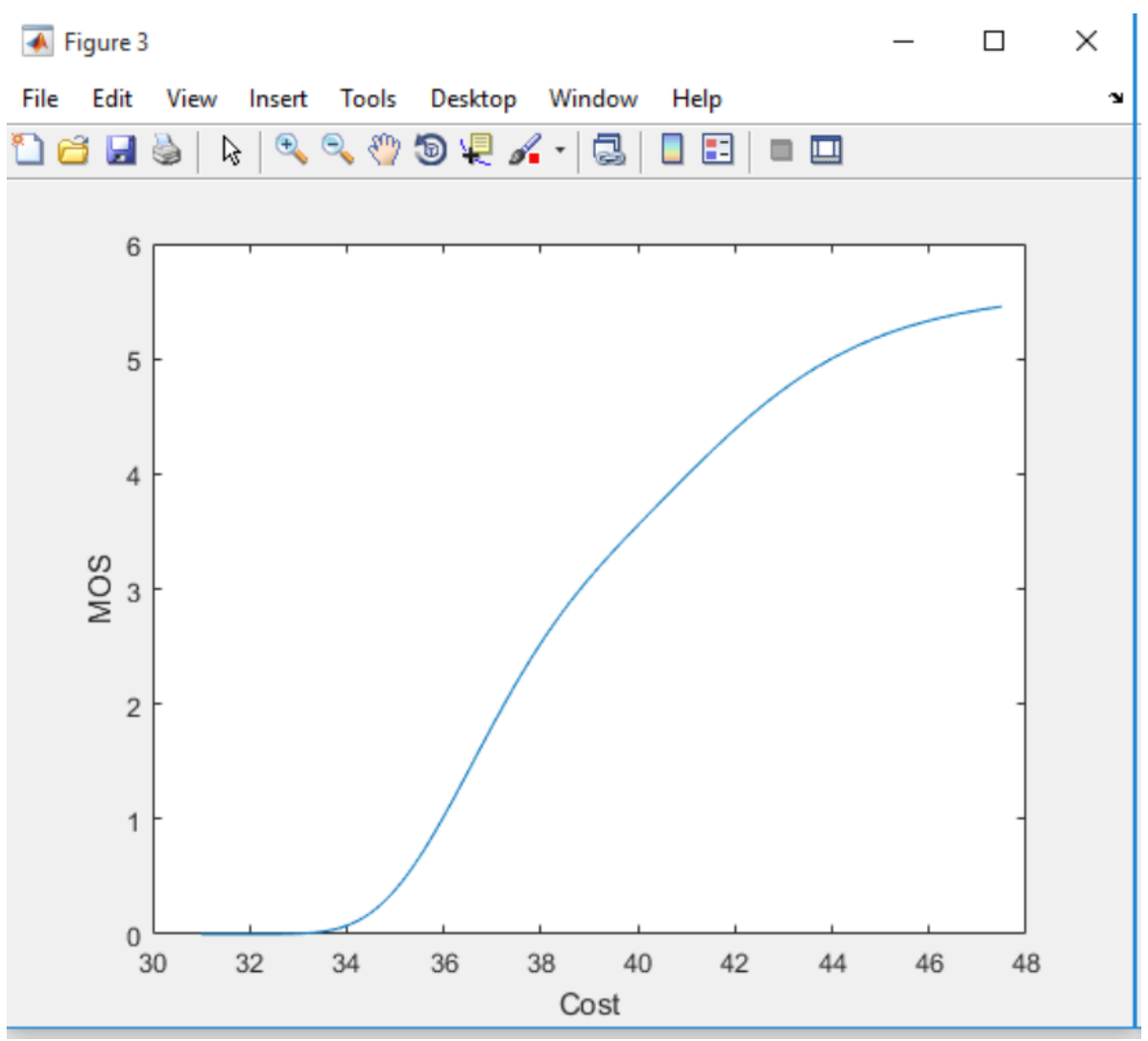

Fig. 6. MOS vs bandwidth cost relationship 


\subsection{Plotting of MOS vs bandwidth cost relationship output}

Initially MoS is very low with low cost. That indicates there is a certain amount of money a customer needs to pay initially to get internet access. A customer paying $38 £$ can obtain quality of experience of 2 MoS. Where a customer paying $45 £$ is receiving far better quality of experience, MoS is close to 5 .

The experiment makes sense if we compare broadband prices in the UK. For example, Broadband provider named 'Now Broadband' offers 11 Mbps in $18 £$ per month in the 'Brilliant Broadband' plan. The Same broadband provider is offering $36 \mathrm{Mbps}$ speed in $24 £$ per month in the 'Fab Fibre' plan. So the bandwidth is increasing more than 3 times while cost is only increasing by $6 £$ (Cable.co.uk, 2019).

\subsection{Plotting of MOS and bandwidth cost Output with different TCP sources}
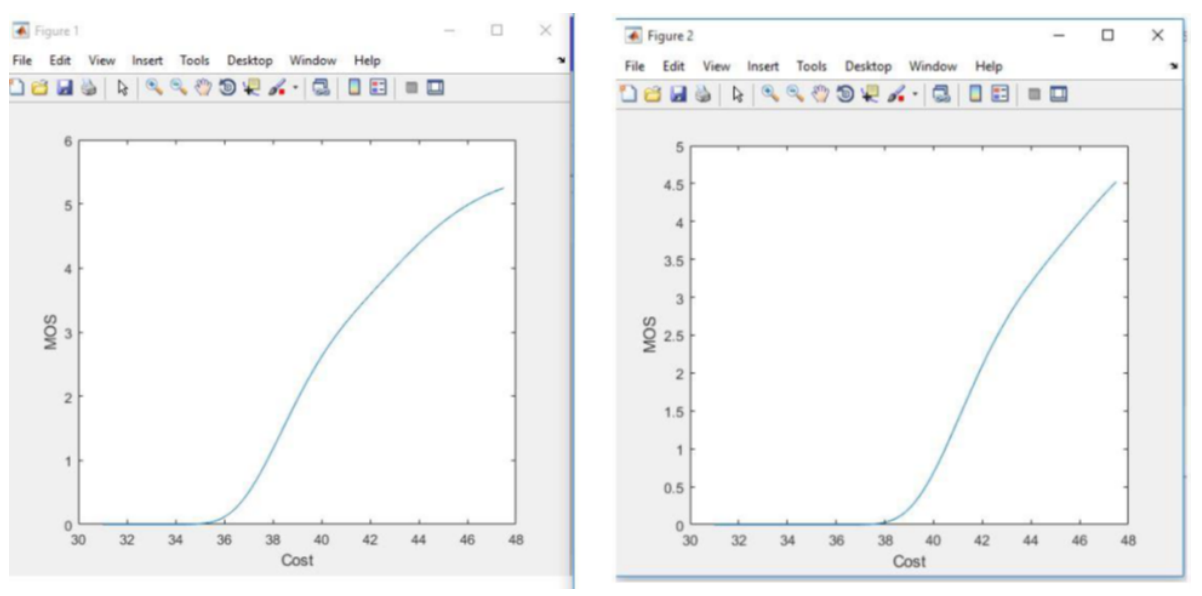

Fig. 7. MOS vs bandwidth Cost Output with different TCP sources.

Initially we took the number of TCP sources to be 50. If we change the TCP sources we obtain different outputs and results. There are 2 output graphs in Fig 7. The first one in Fig(7) is calculated by taking 80 TCP sources and second one is calculated taking $500 \mathrm{TCP}$ sources. If we take more TCP sources the quality increases more rapidly with the price increase compared to taking fewer TCP sources. Big organizations usually have TCP sources and so that they have the luxury of getting better quality of experience within the same cost but after crossing initial price barrier. In small household fewer TCP sources are most likely used which also experiences a rise in MOS which provides good quality within a price range but that is less than that seen in big organizations. 


\section{Discussion and Further Work}

Prior work in the field has shown that QoE has a complex relationship with QoS factors like packet loss probability (PLP), delay or delay jitter. Furthermore, contemporary analyses have indicated that the relationship between these QoS factors and QoE can significantly vary from application to application. In this paper we take prior analyses of the relationship between the key QoS metric of packet loss probability and QoE and target an internet access scenario. We use this relationship to show how QoE (measured as MOS) varies as more money is spent on the bandwidth of the internet access link. Our results target two different scenarios - a small number of multiplexed TCP sources and a relatively large number of multiplexed TCP sources. We show that increase in MOS is not a linear with increasing spent on bandwidth at all, and considering these two different we are able to resolve the highly non-linear fashion in which MOS does increase with spend on bandwidth.

\section{References}

1. Alraddadi, A. (2014). EVALUATION OF PACKET LOSS EFFECT ON NETWORK PERFORMANCE. International Journal of Latest Research in Science and Technology, [online] 3(6), pp.177-182.

2. Aragon, J. (2006). ANALYSIS OF THE CORRELATION BETWEEN PACKET LOSS AND NETWORK DELAY AND THEIR IMPACT IN THE PERFORMANCE OF SURGICAL TRAINING APPLICATIONS. [online]

3. Atwood, J. (2007). The Economics of bandwidth. [Blog] CODING HORROR. Available at: https://blog.codinghorror.com/the-economics-of-bandwidth/ [Accessed 9 Aug. 2019].

4. Bhadra, D., Son, P., Vyas, N., Jhaveri, R. and Joshi, C. (2015). In: IEEE ICCSP. [online]

5. British Telecommunications (2019). Business broadband deals - BT Business. [online] Business.bt.com.

6. Cable.co.uk (2019). Best Broadband Deals August 2019 - Compare Broadband Offers - Cable.co.uk. [online] Cable. Available at: https://www.cable.co.uk/broadband/ [Accessed 16 Aug. 2019].

7. EDUCBA (2018). Uses Of Matlab - Top 15 Best Uses of Matlab In Real Life. [online] EDUCBA.

8. Expert System (2017). What is Machine Learning? A definition - Expert System. [online] Expertsystem.com. Available at: https://www.expertsystem.com/machinelearning-definition/ [Accessed 6 Aug. 2019]. /

9. Gideon, C. (2002). Limits To Certainty in QoS Pricing and bandwidth. [online] Available at: http://hdl.handle.net/1721.1/1512 [Accessed 11 Aug. 2019].

10. Roshan, M., Schormans, J. and Ogilvie, R. (2018). Video-on-demand QoE Evaluation Across Different Age- Groups and Its Significance for Network Capacity. ICST Transactions on Mobile Communications and Applications, [online] 3(9), p.153557.

11. Rouse, M. (2019). Network management and monitoring: The evolution of network control DEFINITION. [online] SearchNetworking. Available at: https://searchnetworking.techtarget.com/definition/round-trip-time [Accessed 11 Aug. 2019]. 
12. Streijl, R., Winkler, S. and Hands, D. (2014). Mean opinion score (MOS) revisited: methods and applications, limitations and alternatives. Multimedia Systems, [online] 22(2), pp.213-227. Available at: https://stefan.winklerbros.net/Publications/mmsj2016.pdf [Accessed 11 Aug. 2019].

13. Techopedia (2017). What is Packet Buffer? - Definition from Techopedia. [online] Techopedia.com. Available at: https://www.techopedia.com/definition/2796/packet-buffer [Accessed 11 Aug. 2019].

14. Techopedia (2017). What is a Network Bottleneck? - Definition from Techopedia. [online] Techopedia.com. Available at: https://www.techopedia.com/definition/24819/networkbottleneck [Accessed 9 Aug. 2019].

15. Walker, D. and Curtis, J. (2019). What is bandwidth?. [online] IT PRO. Available at: https://www.itpro.co.uk/broadband/30274/what-is-bandwidth [Accessed 8 Aug. 2019].

16. Xiao, X. (2008). Technical, commercial and regulatory challenges of QoS. Amsterdam [u.a.]: Elsevier/Morgan Kaufmann, p.30. Zarki, M. (2019). QoS and QoE.

17. Analytical expression and performance evaluation of TCP packet loss probability over geostationary satellite, Publisher: IEEE, Author(s), I. Bisio ; M. Marchese 